\title{
Noninvasive photoacoustic angiography of animal brains in vivo with near-infrared light and an optical contrast agent
}

\author{
Xueding Wang and Geng Ku \\ Optical Imaging Laboratory, Department of Biomedical Engineering, Texas A\&M University, College Station, Texas 77843-3120 \\ Malgorzata A. Wegiel \\ Department of Chemistry and Biochemistry, Texas Tech University, Lubbock, Texas 79409-1061 \\ Darryl J. Bornhop \\ Department of Chemistry, Vanderbilt University, Nashville, Tennessee 37235 \\ George Stoica \\ Department of Pathobiology, Texas A\&M University, College Station, Texas 77843-5547 \\ Lihong V. Wang \\ Optical Imaging Laboratory, Department of Biomedical Engineering, Texas A\&M University, College Station, Texas 77843-3120
}

Received August 21, 2003

\begin{abstract}
Optical contrast agents have been widely applied to enhance the sensitivity and specificity of optical imaging with near-infrared (NIR) light. However, because of the overwhelming scattering of light in biological tissues, the spatial resolution of traditional optical imaging degrades drastically as the imaging depth increases. Here, for the first time to our knowledge, we present noninvasive photoacoustic angiography of animal brains in vivo with NIR light and an optical contrast agent. When indocyanine green polyethylene glycol, a novel absorption dye with prolonged clearance, is injected into the circulatory system of a rat, it obviously enhances the absorption contrast between the blood vessels and the background tissues. Because NIR light can penetrate deep into the brain tissues through the skin and skull, we are able to successfully reconstruct the vascular distribution in the rat brain from the photoacoustic signals. On the basis of differential optical absorption with and without contrast enhancement, a photoacoustic angiograph of a rat brain is acquired that matches the anatomical photograph well and exhibits high spatial resolution and a much-reduced background. This new technology demonstrates the potential for dynamic and molecular biomedical imaging. (C) 2004 Optical Society of America
\end{abstract}

OCIS codes: 170.5120, 170.3880, 170.0110, 170.1470.

The high sensitivity of optical imaging modalities aided by contrast agents parallels that of nuclear imaging and allows the visualization of organs such as the brain without the undesirable effect of ionizing radiation. Indocyanine dyes, with strong absorption in the near-infrared (NIR) spectra, are especially useful because NIR light has low absorption in biological tissues and consequently has relatively deep penetration. For example, indocyanine green (ICG), which has been approved by the Food and Drug Administration, in combination with NIR techniques, is employed widely in clinical applications such as cardiac output monitoring, ${ }^{1}$ hepatic function study, ${ }^{2}$ angiography in ophthalmology ${ }^{3}$ and tumor detection. ${ }^{4}$ However, because of the strong scattering nature of biological tissues in NIR, pure optical imaging techniques, whether diffuse optical tomography, fluorescence imaging, or other microscopies, cannot provide high spatial resolution beyond the skin. The photoacoustic imaging technique, which has the merits of both light and ultrasound, has been proved to be a powerful tool for visualizing biological tissues with satisfactory sensitivity and spatial resolution. ${ }^{5-10}$ In this study, distributions of NIR absorption in biological tissues that were modified by an optical contrast agent were imaged with the photoacoustic modality. With this technique we successfully achieved noninvasive photoacoustic angiography - mapping of the vasculaturein the cortex of rat brains in vivo for what appears to be the first time.

The setup for noninvasive photoacoustic angiography of rat brains is shown in Fig. 1(A). A tunable dye laser (ND6000, Continuum) pumped by a Nd:YAG laser (Brilliant B, Bigsky) was employed to provide laser pulses with a FWHM of $6.5 \mathrm{~ns}$, a pulse repetition rate of $10 \mathrm{~Hz}$, and a wavelength of $805 \mathrm{~nm}$. The incident energy density of the laser beam was controlled to $<2 \mathrm{~mJ} / \mathrm{cm}^{2}$ on the surface of the rat head, which induced a temperature rise in the brain vessels estimated to be $<2.6 \mathrm{mK}$. An unfocused ultrasonic transducer (XMS-310, Panametrics) with a central frequency of $10.4 \mathrm{MHz}$, a bandwidth of $100 \%$ at $-6 \mathrm{~dB}$, and an 


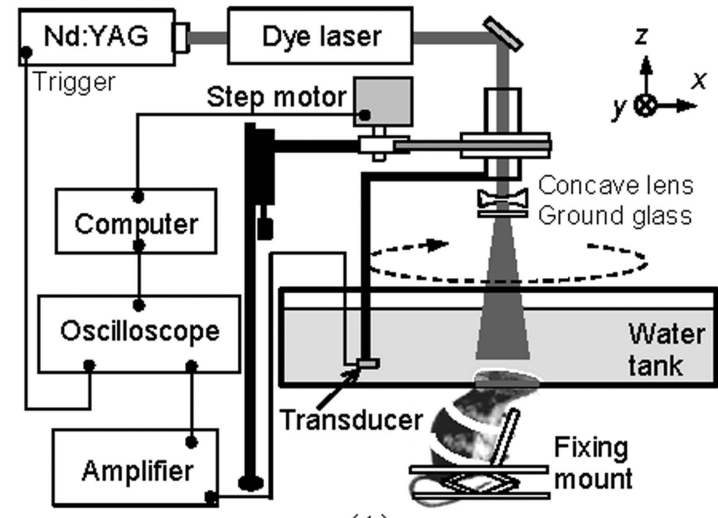

(A)

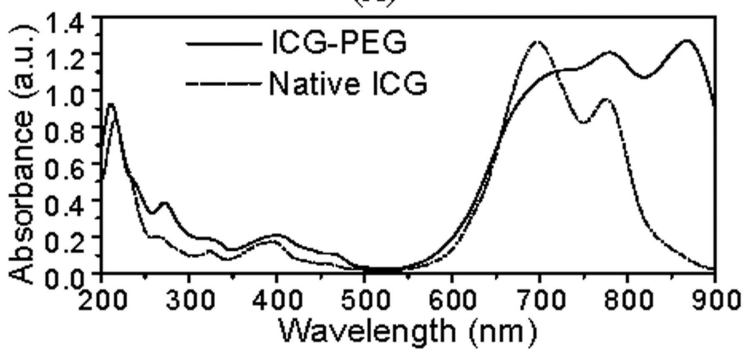

(B)

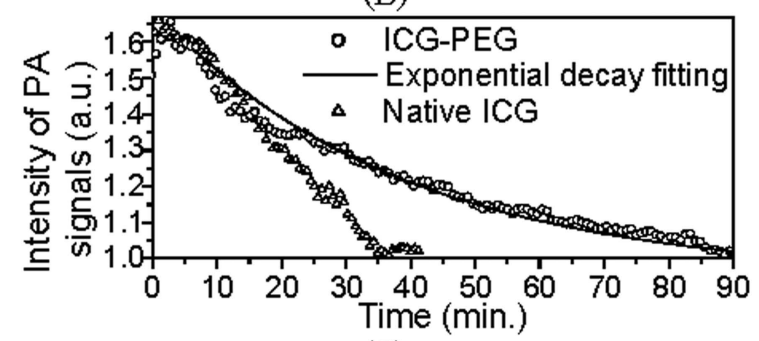

(C)

Fig. 1. (A) Schematic of noninvasive photoacoustic angiography in the rat brain, employing an optical contrast agent and a NIR laser. (B) Absorption spectra of ICG-PEG and native ICG. (C) Intensity of the photoacoustic signals from the blood vessels in the median fissure of the rat brain as a function of time after the intravascular injection of ICG-PEG (circles) or native ICG (triangles), which are normalized to the initial intensity of the photoacoustic signals before the injection. PA, photoacoustic.

active element of $2 \mathrm{~mm}$ in diameter was used to detect the photoacoustic signals. The transducer was driven by a computer-controlled step motor to scan around the cortex of the rat brain in the $x-y$ plane with a radius of $3 \mathrm{~cm}$ and a step size of $1.5^{\circ}$. The data acquisition time for one image was $23.5 \mathrm{~min}$.

The rat was fixed by use of a homemade mount with its head protruding into the water tank through a hole in the bottom of the tank where a piece of clear polyethylene film between the water and the rat head sealed the hole. A thin layer of ultrasonic coupling gel was applied on the surface of the rat head. The photoacoustic signals detected by the transducer were received by an amplifier and then sent to an oscilloscope. Finally, a computer collected the digitized signals to reconstruct the distribution of optical absorption in the imaging plane through a modified backprojection algorithm. ${ }^{11}$ Sprague Dawley rats $(\sim 150$ g, Charles River Breeding Laboratories) were employed for the imaging experiments. Before imaging, the hair on each rat's head was removed with hair remover lotion. A dose of $87 \mathrm{mg} / \mathrm{kg}$ Ketamine plus $13 \mathrm{mg} / \mathrm{kg}$ Xylasine was administered intramuscularly to anesthetize the rats during the data acquisition.

The optical contrast agent was injected into the circulatory system of the rats through the tail vein. To prolong the circulation of ICG in blood, polyethylene glycol (PEG) ${ }^{12}$ a polymer approved by the Food and Drug Administration with the structure $\left(-\mathrm{CH}_{2} \mathrm{CH}_{2} \mathrm{O}-\right)_{n}$, was adopted to stabilize the ICG. This PEG conjugate of ICG (ICG-PEG) with high optical absorption near the $805-\mathrm{nm}$ wavelength [see Fig. 1(B)] was used as a contrast agent in photoacoustic tomography for the first time to our knowledge. The ICG-PEG in phosphate buffered saline $\left(\mathrm{pH}=7.4\right.$; concentration $\left.=3.2 \times 10^{-4} \mathrm{M}\right)$ was injected intravenously at a dosage of $0.25 \mathrm{ml} / 100 \mathrm{~g}$ body weight, which led to an estimated ICG concentration of $\sim 1 \times 10^{-5} \mathrm{M}$ in the blood. With this ICG-PEG dosage and the applied laser energy density for this experiment, the magnitude of the photoacoustic signals received by the ultrasonic transducer was of the order of $10 \mu \mathrm{V}$. Considering the $60-\mu \mathrm{m}$ resolution that has been achieved by this system (to be reported elsewhere), the number of molecules in the $60 \mu \mathrm{m} \times 60 \mu \mathrm{m} \times 60 \mu \mathrm{m}$ resolvable volume is $\sim 2$ fmol, which represents an underestimate of the sensitivity of our imaging system.

By observing the changes in the intensity of the photoacoustic signals from the brain vessels, we studied the time-dependent variation of absorption in the brain blood attributable to the contrast agent [Fig. 1(C)]. The results represent the clearance profile of the dye from the circulatory system of the rat, where ICG-PEG shows an obviously slower clearance than the native ICG.

Photoacoustic angiographs of rat brains in situ based on either the intrinsic contrast or the ICG-PEG enhanced contrast were compared. The contrast between the large blood vessels and the background tissue was approximately 1.8:1 without the contrast agent. The contrast was enhanced to $2.8: 1$ by the ICG-PEG at the applied dosage.

ICG-PEG was applied to noninvasive photoacoustic angiography in the rat brain in vivo (Fig. 2). After the experiment, the rat recovered normally without noticeable health problems. Compared with the reference image in Fig. 2(A), the image in Fig. 2(B), obtained after the injection of ICG-PEG, represents a clearer map of the brain vascular branches that matches well with the photograph of the anatomy. Figure 2(A) was subtracted from the image with contrast enhancement in Fig. 2(B). The differential image in Fig. 2(C) depicts the distribution of differential optical absorption in the rat brain that is attributed to the injected contrast agent. This subtraction reduces the background and further enhances the contrast of blood vessels in rat brains. Some detailed brain vascular structures that cannot be visualized from the image in Fig. 2(B), such as the small vessel branches identified by the black arrows in the figure, were revealed by this differential image. 

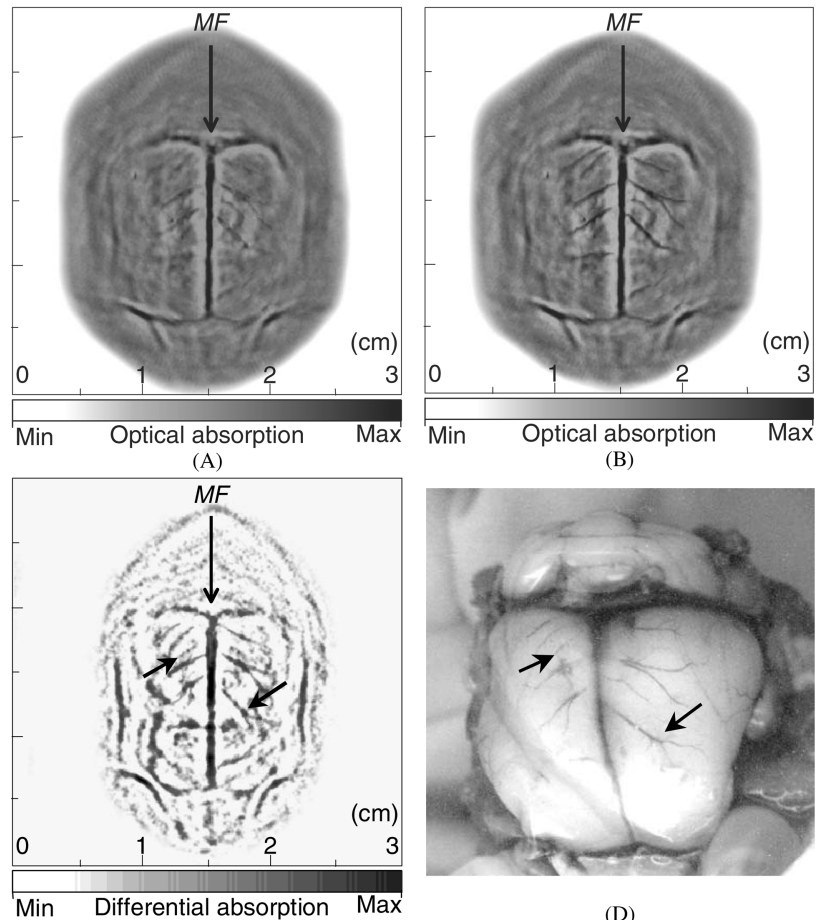

(C)

Fig. 2. Noninvasive photoacoustic images of a rat brain in vivo, employing NIR light and an optical contrast agent (ICG-PEG). (A), (B) Photoacoustic images acquired before and after the injection of ICG-PEG, respectively, where the two gray scales are the same. MF, median fissure. (C) ICG-based angiograph of the rat brain $(\mathrm{C}=\mathrm{B}-\mathrm{A})$. (D) Open-skull photograph of the rat brain obtained after the data acquisition for photoacoustic imaging.

More important, this experiment demonstrated the potential of photoacoustic tomography for dynamic and molecular imaging.

A circular scan of even an unfocused transducer was able to produce high-quality images of the cerebral cortex for the following reasons. Most large blood vessels are distributed in the brain cortex. The photoacoustic signals that originated from the strong optical absorption in the vasculature of the dorsal cerebral cortex were dominant. Conversely, the photoacoustic signals from the large vessels in the ventral brain were relatively small because of the attenuation of light in the brain. In addition, the aperture effect of the ultrasonic transducer provided some resolution along the $z$ axis.

Images obtained with this setup represent a distribution of the averaged optical absorption during the data acquisition. In this case the applied contrast agent with a prolonged clearance time benefits the acquisition of imaging signals, especially when several images need to be acquired with the current imaging system after the intravascular injection. When an ultrasonic transducer array, instead of a singleelement transducer, is adopted in the future, this technology will provide real-time and more accurate

quantitative monitoring of the brain hemodynamics. Consequently, the clearance time will not need to be prolonged in that case. The in vivo dynamics of the exogenous dye can provide useful physiological information.

In summary, angiography in the rat brain yielding high contrast and high spatial resolution based on the photoacoustic imaging of exogenous contrast agents has been implemented noninvasively. This technique provides an accurate noninvasive monitoring method for fluid pathways in biological tissues, which makes it a powerful method for imaging vascular changes in tumors, delineating neovascularization, and studying global and regional hemodynamic activities in the brain. Since the contrast agent is expected to accumulate in neoplastic tissues or traumatized regions, this technique is promising for determining the margins of embedded tumors or bruises with a high degree of accuracy. More important, since contrast agents can be conjugated to bioactive peptides, proteins, antibodies, hormones, drugs, or other bioactive agents, this technique can be readily extended to molecular and functional imaging. For example, with highfrequency ultrasonic detection and tumor-targeted optical contrast agents, this technique offers promise for imaging pathologic processes at molecular and genetic levels. All these objectives can be accomplished by virtue of the high optical contrast and high ultrasonic resolution that this technique provides.

This study was sponsored in part by the National Institutes of Health, the Department of Defense, the National Science Foundation, and the Texas Advanced Research Program. L. Wang's e-mail address is LWang@tamu.edu.

\section{References}

1. Y.-L. He, H. Tanigami, H. Ueyama, T. Mashimo, and I. Yoshiya, Crit. Care Med. 26, 1446 (1998).

2. J. Caesar, S. Shaldon, L. Chiandussi, L. Guevara, and S. Sherlock, Clin. Sci. 21, 43 (1961).

3. B. F. Hochheim, Arch. Ophthalmol. 86, 564 (1971).

4. M. M. Haglund, M. S. Berger, and D. W. Hochman, Neurosurgery 38, 308 (1996).

5. C. G. A. Hoelen, F. F. M. de Mul, R. Pongers, and A. Dekker, Opt. Lett. 23, 648 (1998).

6. R. A. Kruger, D. R. Reinecke, and G. A. Kruger, Med. Phys. 26, 1832 (1999).

7. R. O. Esenaliev, A. A. Karabutov, and A. A. Oraevsky, IEEE J. Sel. Top. Quantum Electron. 5, 981 (1999).

8. K. P. Köstli, D. Frauchiger, J. J. Niederhauser, G. Paltauf, H. P. Weber, and M. Frenz, IEEE J. Sel. Top. Quantum Electron. 7, 918 (2001).

9. X. Wang, Y. Pang, G. Ku, X. Xie, G. Stoica, and L. V. Wang, Nat. Biotechnol. 21, 803 (2003).

10. X. Wang, Y. Pang, G. Ku, G. Stoica, and L. V. Wang, Opt. Lett. 28, 1739 (2003).

11. M. Xu and L. V. Wang, IEEE Trans. Med. Imaging 21, 814 (2002).

12. S. Zalipsky, E. Brandeis, M. S. Newman, and M. C. Woodle, FEBS Lett. 353, 71 (1994). 